\title{
Self-organized criticality in X-ray flares of gamma-ray-burst afterglows
}

\author{
F. Y. Wang ${ }^{1,2 \star}$ and Z. G. Dai ${ }^{1,2 \star}$
}

X-ray flares detected in nearly half of gamma-ray-burst (GRB) afterglows are one of the most intriguing phenomena in highenergy astrophysics ${ }^{1-8}$. All of the observations indicate that the central engines of bursts, after the gamma-ray emission has ended, still have long periods of activity, during which energetic explosions eject relativistic materials, leading to late-time $X$-ray emission ${ }^{2,9,10}$. It is thus expected that $X$-ray flares provide important clues as to the nature of the central engines of GRBs, and more importantly, unveil the physical mechanism of the flares themselves, which has so far remained mysterious. Here we report statistical results of $X$-ray flares of GRBs with known redshifts, and show that X-ray flares and solar flares share three statistical properties: power-law frequency distributions for energies, durations and waiting times. All of the distributions can be well understood within the physical framework of a self-organized criticality (SOC) system. The statistical properties of X-ray flares of GRBs are similar to solar flares, and thus both can be attributed to a SOC process. Both types of flares may be driven by a magnetic reconnection process, but X-ray flares of GRBs are produced in ultra-strongly magnetized millisecond pulsars ${ }^{11,12}$ or long-term hyperaccreting disks around stellar-mass black holes ${ }^{13}$.

GRBs are flashes of gamma-rays occurring at cosmological distances with an isotropic-equivalent energy release from $10^{51}$ to $10^{54} \mathrm{ergs}^{9,10,14,15}$. They can be sorted into two classes: shortduration hard-spectrum bursts $(<2 s)$ and long-duration softspectrum bursts ${ }^{16}$. Thanks to the rapid-response capability and high sensitivity of the Swift satellite ${ }^{17}$, numerous unforeseen features have been discovered, one of which is that about half of the bursts have large, late-time X-ray flares with short rise and decay times ${ }^{4,5}$. Unexpected X-ray flares with an isotropicequivalent energy from $10^{48}$ to $10^{52}$ ergs have been detected for both long and short bursts ${ }^{4,6,7}$. The occurrence times of X-ray flares range from a few seconds to $10^{6}$ seconds after the GRB trigger ${ }^{8}$. Until now, the physical mechanism of X-ray flares has remained mysterious, although some models have been proposed ${ }^{9,10}$. Due to the 8-year observations of Swift, plentiful X-ray flare data have been collected. Here we investigate the frequency distributions of energies, durations and waiting times of GRB X-ray flares for the first time. On the other hand, it is well known that solar flares with a timescale of hours are explosive phenomena in the solar atmosphere with an energy release of about $10^{28}-10^{32}$ ergs, which are widely believed to be triggered by a magnetic reconnection process ${ }^{18}$. They have been observed in broadband electromagnetic waves, but we focus here on solar hard X-ray flares.

Although X-ray flares are common phenomena in GRBs and the Sun, the flare energy spans about 20 orders of magnitude and an outstanding question appears, namely, do GRB X-ray flares and solar flares have a similar physical mechanism? Interestingly, some theoretical models have suggested that GRB X-ray flares could be magnetically dominated explosive events ${ }^{11-13}$. However, a physical analogy between GRB X-ray flares and solar flares has not yet been established.

We search for statistical similarities between GRB X-ray flares and solar flares. In particular, we compare the statistical properties of the energy release, duration-time and waiting-time distributions of GRB X-ray flares and solar flares. For X-ray flares of GRBs with known redshifts, we employ published and archival observed data that allow us to estimate the energy release, duration time and waiting time of each $\mathrm{X}$-ray flare $\mathrm{e}^{-8}$. The total number of flares is 83 , including 9 short-burst flares and 74 long-burst flares. The isotropic energy of one flare in the $0.3-10 \mathrm{keV}$ band can be calculated by $E_{\text {iso }}=4 \pi d_{\mathrm{L}}^{2}(z) S_{\mathrm{F}} /(1+z)$, where $S_{\mathrm{F}}$ is the fluence, $z$ is the redshift and $d_{\mathrm{L}}(z)$ is the luminosity distance calculated for a flat cosmological-constant cold dark matter universe with $\Omega_{\mathrm{M}}=0.3$, $\Omega_{\Lambda}=0.7$ and $H_{0}=70 \mathrm{~km} \mathrm{~s}^{-1} \mathrm{Mpc}^{-3}$. The Malmquist bias refers to an effect that leads to the preferential detection of intrinsically bright objects, but this effect is claimed to be small and negligible for GRBs spreading over a wide range of redshift ${ }^{19}$. The waiting time in the source's rest frame can be obtained by $\Delta t=\left(t_{i+1}-t_{i}\right) /(1+z)$, where $t_{i+1}$ is the observed starting time of the $i+1$ th flare, $t_{i}$ is the observed starting time of the $i$ th flare, and $(1+z)$ is the relativistic time dilation factor to transfer the time into the source's rest frame. For the first flare appearing in an afterglow, the waiting time is taken to be $t_{1} /(1+z)$. We list the measured parameters of the $83 \mathrm{X}$-ray flares in Supplementary Table S1.

Figure 1 shows the cumulative energy distribution of GRB $\mathrm{X}$-ray flares and the differential energy frequency distribution of solar hard X-ray flares. If the number of events $N(E) \mathrm{d} E$ with energy between $E$ and $E+\mathrm{d} E$ obeys a power-law relation, $N(E) \mathrm{d} E \propto E^{-\alpha_{E}} \mathrm{~d} E$ for $E<E_{\max }$, with index of $\alpha_{E}$ and a cutoff energy of $E_{\max }$, then we can calculate the number of events with energy larger than $E$ through $N(>E)=a+b\left[E^{1-\alpha_{E}}-E_{\max }^{1-\alpha_{E}}\right]$, where $a$ and $b$ are two parameters. To obtain the best-fitting parameters, the Markov chain Monte Carlo technique is used in our calculations. We obtain $\alpha_{E} \simeq 1.06 \pm 0.15$ from the cumulative energy distribution for GRB X-ray flares. Furthermore, the blue and green curves in Fig. 1 represent the differential energy frequency distribution with $\alpha_{E}=1.65 \pm 0.02$ for Ramaty High Energy Solar Spectroscopic Imager (RHESSI) solar flares ${ }^{20}$, and $\alpha_{E}=1.53 \pm$ 0.02 for Hard X-ray Burst Spectrometer (HXRBS) solar flares ${ }^{21}$, respectively. The energy of a solar flare is the non-thermal energy in electrons above $25 \mathrm{keV}$. A distinct difference in the energy release distributions between GRB X-ray flares and solar flares is that the former's high-energy cutoff is $E_{\max } \approx 2 \times 10^{52}$ ergs. In Fig. 2, we present the duration-time $(T)$ frequency distributions of solar flares and GRB X-ray flares, which can also be fitted by a power-law relation with an index of $\alpha_{T}$, that is $N(T) \mathrm{d} T \propto T^{-\alpha_{T}} \mathrm{~d} T$. The red

${ }^{1}$ School of Astronomy and Space Science, Nanjing University, Nanjing 210093, China, ${ }^{2}$ Key Laboratory of Modern Astronomy and Astrophysics (Nanjing University), Ministry of Education, Nanjing 210093, China. `e-mail: fayinwang@nju.edu.cn; dzg@nju.edu.cn 

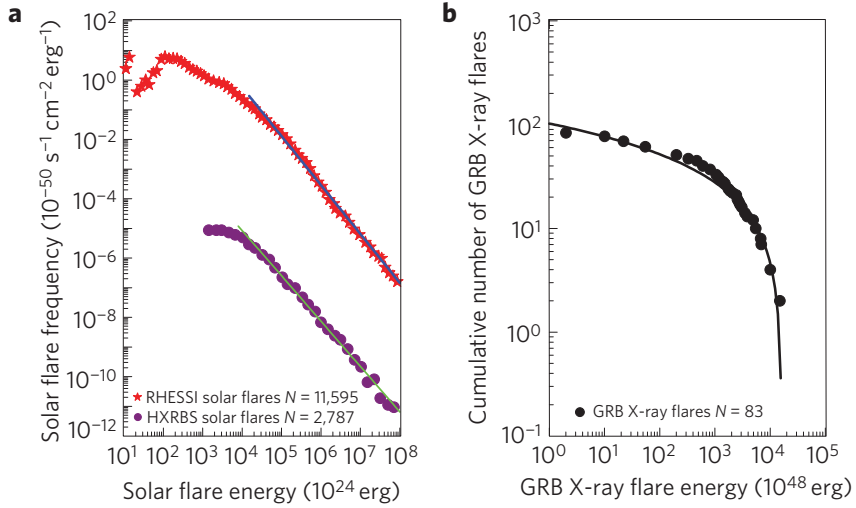

Figure 1 | Energy-release frequency distributions. a, The differential energy frequency distribution of solar hard X-ray flares. We employ 11,595 solar flares ${ }^{20}$ from RHESSI during 2002-2007, and 2,787 flares ${ }^{21}$ from HXRBS during 1980-1982. For a differential frequency distribution $N(E) \mathrm{d} E \propto E^{-\alpha_{E}} \mathrm{~d} E$ of solar flares, the blue and green curves give $\alpha_{E}=1.65 \pm 0.02$ for the RHESSI sample, and $1.53 \pm 0.02$ for the HXRBS sample, respectively. $\mathbf{b}$, The cumulative energy distribution of GRB X-ray flares. The black curve gives the cumulative energy distribution $N(>E)=a+b\left[E^{1-\alpha_{E}}-E_{\max }^{1-\alpha_{E}}\right]$, with $\alpha_{E} \sim 1.06 \pm 0.15$ for GRB X-ray flares. The SOC theory predicts that the value of $\alpha_{E}$ is 1.0 for one dimension, and 1.5 for three dimensions, respectively ${ }^{22}$. Although the power-law indices are apparently different for both types of flares, their statistical properties can be explained within the same framework of a SOC system, as discussed in the text.

lines in Fig. 2 show a power-law fit with $\alpha_{T}=1.10 \pm 0.15$ for GRB $\mathrm{X}$-ray flares, and $2.00 \pm 0.05$ for solar flares, respectively.

Although the energy and duration-time frequency distributions for both kinds of flares are apparently different, we next show that these distributions can be well understood within the same statistical framework of a SOC system. The energy and duration frequency distributions of solar fares have been thought to be attributed to a magnetic reconnection process on the basis of the fractal-diffusive avalanche mode ${ }^{20,22,23}$. We further discuss this model to explain the energy and duration-time frequency distributions of GRB X-ray flares. For a SOC system ${ }^{24}$, owing to some driving force, subsystems will self-organize to a critical state at which a small perturbation can trigger an avalanche-like chain reaction of any size within the system. Due to diffuse random walking, a statistical relationship ${ }^{22}$ between the size scale $L$ and the duration time $T$ of a SOC avalanche is $L \propto T^{1 / 2}$, and a probability distribution of size $L$ is argued as $N(L) \mathrm{d} L \propto L^{-S} \mathrm{~d} L$ for the three Euclidean dimensions $S=1,2$ and 3 . This probability argument is based on the assumption that the occurrence frequency or number of events is equally likely throughout the system. So the index of the duration frequency distribution of flares is given by $^{22}$

$$
\alpha_{T}=\frac{S+1}{2}
$$

This index becomes $\alpha_{T}=1$ for $S=1$ and $\alpha_{T}=2$ for $S=3$, which can well explain the observed duration distributions of GRB X-ray flares and solar flares. On the other hand, the index of the energy frequency distribution can be written a $^{22}$

$$
\alpha_{E}=\frac{3(S+1)}{S+5}
$$

It is easy to see that the index $\alpha_{E}=1$ for $S=1$ and $\alpha_{E}=1.5$ for $S=3$, which are remarkably consistent with the observed indices of GRB $\mathrm{X}$-ray flares and solar flares. Interestingly, soft $\gamma$-ray repeaters also
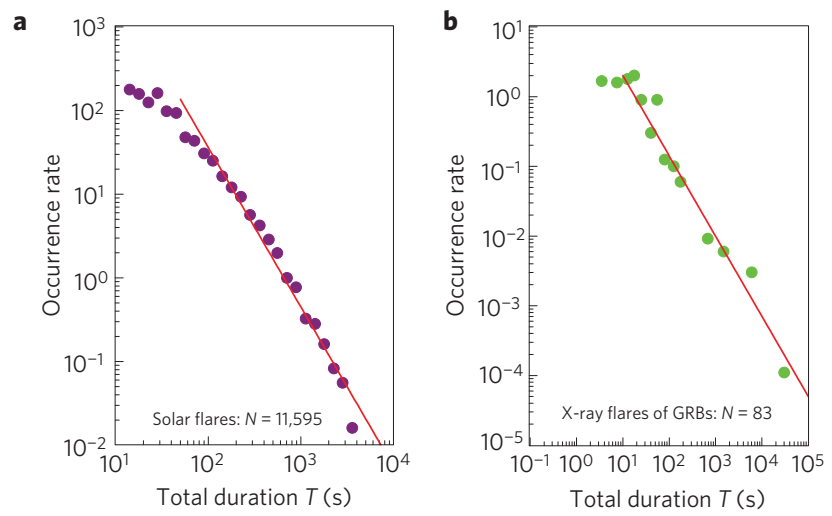

Figure 2 | Duration-time frequency distributions. a, The relation between the occurrence rate and duration time for solar flares. The occurrence rate is defined by the ratio of the number of $X$-ray flares in the bin to the bin width. The total duration times of 11,595 solar flares ${ }^{20}$ were observed by RHESSI during 2002-2007. The best-fit power-law index is $-2.00 \pm 0.05$. b. The relation between the occurrence rate and duration time for GRB $\mathrm{X}$-ray flares. The best-fit power-law index is $-1.10 \pm 0.15$.

have a power-law energy frequency distribution ${ }^{25,26}$ with $\alpha_{E} \sim 1.5$. A power-law distribution of occurrence frequency is characteristic of a SOC system ${ }^{20}$. According to equations (1) and (2), the power-law indices of the energy and duration-time distributions of SOC depend on the Euclidean space dimensions $S$ of the energy dissipation domain $^{22}$. Thus, it is clear that GRB X-ray flares and solar flares correspond to the one-dimensional $(S=1)$ and three-dimensional $(S=3)$ cases, respectively. Therefore, GRB X-ray flares and solar flares share energy and duration-time frequency distributions, suggesting that they can be explained with the same statistical framework of SOC systems, but with different Euclidean dimensions.

Now we discuss the waiting-time distributions for both kinds of flares. The waiting time is defined as the time interval between two successive events. Its distribution gives us information on whether events occur independently, and provides the mean rate of event occurrence ${ }^{20}$. It has been suggested that the waitingtime distribution of solar flares can be described by a power-law distribution with an index of about -2.0 for long waiting times ${ }^{27,28}$. But the waiting-time distribution of GRB X-ray flares has not been studied previously. Figure 3 shows the waiting-time distribution of GRB X-ray flares and solar flares. Excluding the fluctuations of short waiting times, the waiting-time distribution of GRB X-ray flares is also a power-law with index $-1.80 \pm 0.20$. The solar flares with waiting times larger than about $2 \mathrm{~h}$ observed by RHESSI during 2002-2009 can be fitted by a power-law function with an index of $-2.0 \pm 0.05$. Thus, GRB X-ray flares and solar flares have similar waiting-time distributions, which can be explained by non-stationary Poisson processes ${ }^{27}$. A Poissonian random process has an exponential waiting-time distribution for a stationary flare rate and a power-law-like waiting-time distribution for a nonstationary flare rate, which is the prediction of the SOC theory ${ }^{20}$. For a non-stationary Poisson process, the waiting-time distribution can be expressed by ${ }^{27}$

$$
P(\Delta t)=\frac{\lambda_{0}}{\left(1+\lambda_{0} \Delta t\right)^{2}}
$$

where $\lambda_{0}$ is the mean rate of flares. For large waiting times $\left(\Delta t \gg 1 / \lambda_{0}\right)$, equation (3) approaches a power-law relation $P(\Delta t) \approx\left(1 / \lambda_{0}\right)(\Delta t)^{-2}$, which is consistent with the observations. We can see from Fig. 3 that the breakpoint is around $\Delta t_{0}=1 / \lambda_{0} \sim 20 \mathrm{~s}$ in the source's rest frame for GRB X-ray flares (so the mean rate is $\lambda_{0} \sim 0.05 \mathrm{~s}^{-1}$ ), and $\Delta t_{0} \sim 1.2 \mathrm{~h}$ for solar flares. 

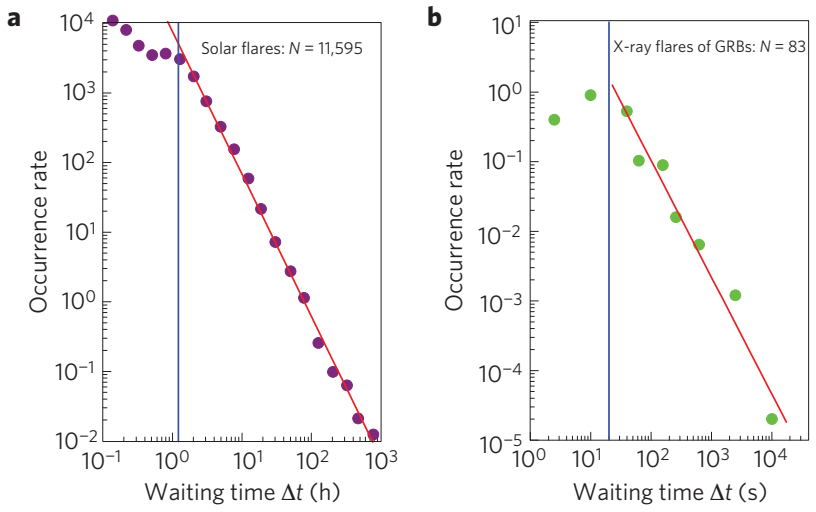

Figure $\mathbf{3}$ | Waiting-time frequency distributions. a, The relation between the occurrence rate and waiting time for solar flares. The waiting times of 11,595 solar flares ${ }^{27}$ were observed by RHESSI during 2002-2007. The best-fit power-law index is $-2.04 \pm 0.03$. The blue vertical line shows the waiting time at the breakpoint, corresponding to the mean rate of flares. The waiting time at the breakpoint is about $1.2 \mathrm{~h}$ for solar flares. $\mathbf{b}$, The relation between the occurrence rate and waiting time for GRB X-ray flares. The best-fit power-law index is $-1.80 \pm 0.20$. The waiting time at the breakpoint is about $20 \mathrm{~s}$ in the source's rest frame for GRB X-ray flares.

In short, we find that GRB X-ray flares and solar flares have similar statistical properties but different Euclidean dimensions of SOC systems. Which models for X-ray flares are consistent with an $S=1$ SOC process? The observations indicate that the central engines of GRBs have long-lasting activity and X-ray flares arise from late internal shocks, which could be formed through collisions of relativistic shells ejected after the prompt gamma-ray emission has ended ${ }^{2,9,10}$. Such shells could be powered by differentially rotating, ultra-strongly magnetized, millisecond pulsars ${ }^{11}$. The differential rotation leads to windup of poloidal magnetic fields in the interior and the resulting toroidal fields are strong enough to float up and break through the stellar surface ${ }^{29,30}$. Magnetic reconnection-driven multiple explosions then occur, producing X-ray flares. Some internal dissipations of relativistic winds from postburst millisecond magnetars (a type of pulsar with a magnetic field strength of $\sim 10^{14}-10^{15}$ Gauss) could also lead to X-ray flares ${ }^{12}$. Furthermore, relativistic shells could be ejected from long-term hyperaccreting disks around stellar-mass black holes by some mechanism, for example the magnetic barrier effect ${ }^{13}$. It is generally believed that the magnetic fields in the shells produced in these models will be mainly transverse, because any radial component decays faster with radius. Thus, the magnetic fields in the shells are stronger compared to matter kinetic energies, and hence closer to one dimension rather than the three dimensions for the much weak solar fields. This can provide an explanation of why the $\alpha_{E}$ and $\alpha_{T}$ indices of GRB X-ray flares are different from those of solar flares, as we found above.

Received 7 April 2013; accepted 23 May 2013; published online 2 July 2013

\section{References}

1. Piro, L. et al. Probing the environment in gamma-ray bursts: The case of an $\mathrm{X}$-ray precursor, afterglow late onset, and wind versus constant density profile in GRB 011121 and GRB 011211. Astrophys. J. 623, 314-324 (2005).

2. Burrows, D. N. et al. Bright X-ray flares in gamma-ray burst afterglows. Science 309, 1833-1835 (2005).

3. Barthelmy, S. D. et al. An origin for short $\gamma$-ray bursts unassociated with current star formation. Nature 438, 994-996 (2005).

4. Falcone, A. D. et al. The first survey of X-ray flares from gamma-ray bursts observed by Swift: Spectral properties and energetics. Astrophys. J. 671, 1921-1938 (2007).
5. Chincarini, C. et al. The first survey of x-ray flares from gamma-ray bursts observed by Swift: Temporal properties and morphology. Astrophys. J. 671, 1903-1920 (2007).

6. Chincarini, C. et al. Unveiling the origin of X-ray flares in gamma-ray bursts. Mon. Not. R. Astron. Soc. 406, 2113-2148 (2010).

7. Margutti, R. et al. X-ray flare candidates in short gamma-ray bursts. Mon. Not. R. Astron. Soc. 417, 2144-2160 (2011).

8. Bernardini, M. G., Margutti, R., Chincarini, C., Guidorzi, C. \& Mao, J. Gamma-ray burst long lasting X-ray flaring activity. Astron. Astrophys. 526, A27 (2011).

9. Mészáros, P. Gamma-Ray Bursts. Rep. Prog. Phys. 69, 2259-2322 (2006).

10. Zhang, B. Gamma-ray bursts in the Swift era. Chin. J. Astron. Astrophys. 7, 1-50 (2007).

11. Dai, Z. G., Wang, X. Y., Wu, X. F. \& Zhang, B. X-ray flares from postmerger millisecond pulsars. Science 311, 1127-1129 (2006).

12. Metzger, B. D., Giannios, D., Thompson, T. A., Bucciantini, N. \& Quataert, E. The protomagnetar model for gamma-ray bursts. Mon. Not. R. Astron. Soc. 413, 2031-2056 (2011).

13. Proga, D. \& Zhang, B. The late time evolution of gamma-ray bursts: Ending hyperaccretion and producing flares. Mon. Not. R. Astron. Soc. 370, L61-L65 (2006).

14. Piran, T. The physics of gamma-ray bursts. Rev. Mod. Phys. 76, 1143-1210 (2004)

15. Gehrels, N., Ramirez-Ruiz, E. \& Fox, D. B. Gamma-ray bursts in the Swift era. Ann. Rev. Astron. Astrophys. 47, 567-617 (2009).

16. Kouveliotou, C. et al. Identification of two classes of gamma-ray bursts. Astrophys. J. 413, L101-L104 (1993).

17. Gehrels, N. et al. The Swift gamma-ray burst mission. Astrophys. J. 611, 1005-1020 (2004)

18. Shibata, K. \& Magara, T. Solar flares: Magnetohydrodynamic processes. Living Rev. Solar Phys. 8, 6-104 (2011).

19. Schaefer, B. E. The Hubble diagram to redshift $>6$ from 69 gamma-ray bursts. Astrophys. J. 660, 16-46 (2007).

20. Aschwanden, M. J. Self-organized Criticality in Astrophysics: The Statistics of Nonlinear Processes in the Universe (Springer, 2011).

21. Crosby, N. B., Aschwanden, M. J. \& Dennis, B. R. Frequency distributions and correlations of solar X-ray flare parameters. Solar Phys. 143, 275-299 (1993).

22. Aschwanden, M. J. A statistical fractal-diffusive avalanche model of a slowly-driven self-organized criticality system. Astron. Astrophys. 539, A2 (2012).

23. Lu, E. T. \& Hamilton, R. J. Avalanches and the distribution of solar flares. Astrophys. J. 380, L89-L92 (1991).

24. Bak, P., Tang, C. \& Wiesenfeld, K. Self-organized criticality: An explanation of the 1/f noise. Phys. Rev. Lett. 59, 381-384 (1987).

25. Gögüuş, E et al. Statistical properties of SGR 1900+14 bursts. Astrophys. J. 526, L93-L96 (1999).

26. Gögüus, E. et al. Statistical properties of SGR 1806-20 bursts. Astrophys. J. 532, L121-L124 (2000).

27. Aschwanden, M. J. \& McTiernan, J. M. Reconciliation of waiting time statistics of solar flares observed in hard X-rays. Astrophys. J. 717, 683-692 (2010).

28. Wheatland, M. S., Sturrock, P. A. \& McTiernan, J. M. The waiting-time distribution of solar flare hard X-ray bursts. Astrophys. J. 509, 448-455 (1998).

29. Kluźniak, W. \& Ruderman, M. The central engine of gamma-ray bursters. Astrophys. J. 505, L113-L117 (1998).

30. Dai, Z. G. \& Lu, T. $\gamma$-ray bursts and afterglows from rotating strange stars and neutron stars. Phys. Rev. Lett. 81, 4301-4304 (1998).

\section{Acknowledgements}

We thank M. J. Aschwanden, P. F. Chen, Y. Dai, M. D. Ding, Y. Guo, Y. F. Huang and X. Y. Wang for discussions. This work was supported by the National Natural Science Foundation of China (grant Nos 11103007 and 11033002).

\section{Author contributions}

F.Y.W. analysed the observational data and explained the statistical results based on a self-organized criticality theory. Z.G.D. suggested such an analysis and a comparison of GRB X-ray flares with solar flares, and proposed the physical mechanism of GRB X-ray flares. Both authors wrote and discussed the manuscript together.

\section{Additional information}

Supplementary information is available in the online version of the paper. Reprints and permissions information is available online at www.nature.com/reprints.

Correspondence and requests for materials should be addressed to F.Y.W. or Z.G.D.

\section{Competing financial interests}

The authors declare no competing financial interests. 